\title{
Active earth pressure model tests versus finite element analysis
}

\author{
Magdalena Pietrzak* \\ Koszalin University of Technology, Department of Civil, Geodetic and Environmental Science, 74-453 Koszalin, Poland
}

\begin{abstract}
The purpose of the paper is to compare failure mechanisms observed in small scale model tests on granular sample in active state, and simulated by finite element method (FEM) using Plaxis 2D software. Small scale model tests were performed on rectangular granular sample retained by a rigid wall. Deformation of the sample resulted from simple wall translation in the direction 'from the soil" (active earth pressure state. Simple Coulomb-Mohr model for soil can be helpful in interpreting experimental findings in case of granular materials. It was found that the general alignment of strain localization pattern (failure mechanism) may belong to macro scale features and be dominated by a test boundary conditions rather than the nature of the granular sample.
\end{abstract}

\section{Introduction}

The purpose of the paper is to compare failure mechanisms observed in small scale model tests on granular sample in active state, and simulated by finite element method (FEM) using Plaxis 2D software.

Experimental failure mechanisms were obtained by image analysis (particle image velocimetry PIV, geoPIV_RG software). Failure mechanism in a granular material consists of a system of localised deformation (shear bands) best visible on shear strain maps which can be calculated from displacement fields (Fig.2A, Fig.3 Experiment).

The corresponding mechanisms produced by numerical simulation (FEM) are best visible on maps of shear strain increments (Fig.2B, C, Fig.3 Calculations).

\subsection{PIV method}

Particle image velocimetry is a well-known velocity measuring non-invasive procedure, originally developed for fluid mechanics and used for the analysis of displacements in tests on soil models.

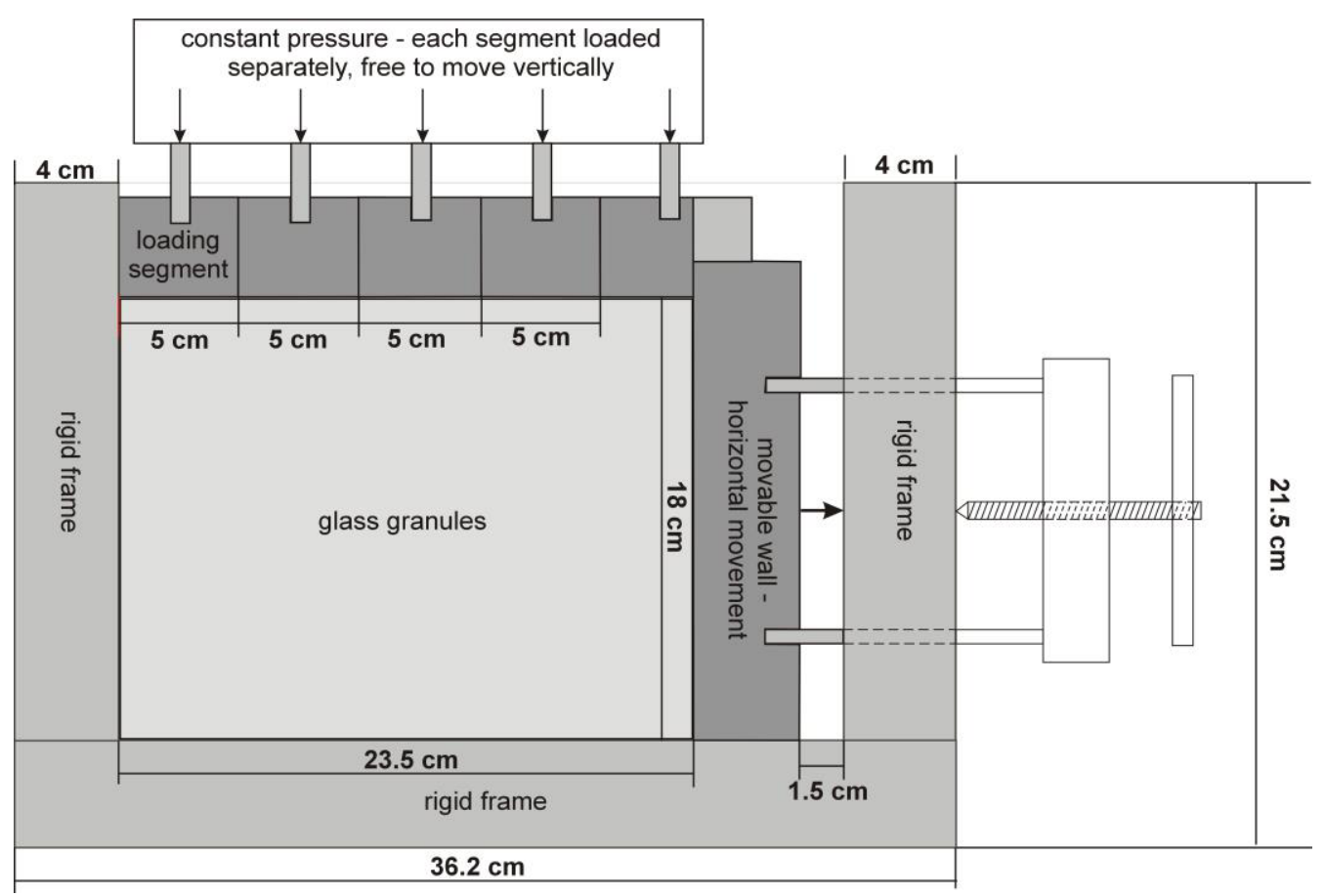

Fig. 1. Experimental setup.

\footnotetext{
* Corresponding author: magdalena.pietrzak@tu.koszalin.pl
} 
It operates by tracking spatial variations of brightness within an image (divided into a mesh of PIV patches) by comparing successive images so that displacement data can be extracted from sequences of images, and strains then calculated from gradients of measured displacements, [1-3].

The geoPIV_RG software developed by White and Take [1-3] for granular materials was employed in this study.

The first step in geoPIV_RG application is to divide the digital image into square patches of pixels forming a regular mesh - the extremes of the mesh coarseness were discussed by Leśniewska and Muir Wood in [4].

According to White et al. [3], the precision of PIV is a strong function of the patch size that is to be compared from image to image and is also influenced by the image content. If the patch is too small, then the software may not be able to recognize the displaced patch. A finer grid provides more details but also a greater potential for random data fluctuations. A trial and error method indicated that a reliable result (a displacement field containing no spurious vectors) could be obtained by using the mesh of square $40 \times 40$ pixel patches.

\subsection{Finite element method - Plaxis software}

Finite element method is based on formalism of the mechanics of continuous media [5]. Plaxis 2D is mainly used for analysis of boundary problems in the field of soil mechanics and geotechnics.

Numerical analysis by Plaxis in this paper consisted of simulation of the model tests' initial geometry and then the granular sample deformation under applied loads.

15 nodes triangular elements were employed in the analysis, containing 3 external and 12 internal nodes.

Results of finite element analysis depend on many factors, such as adopted constitutive models of materials, values of material parameters, boundary conditions. Plaxis contains a number of constitutive models, from which the linear elastic model is most often applied for structural elements, and elasto-plastic Mohr-Coulomb model and its extensions for soils.

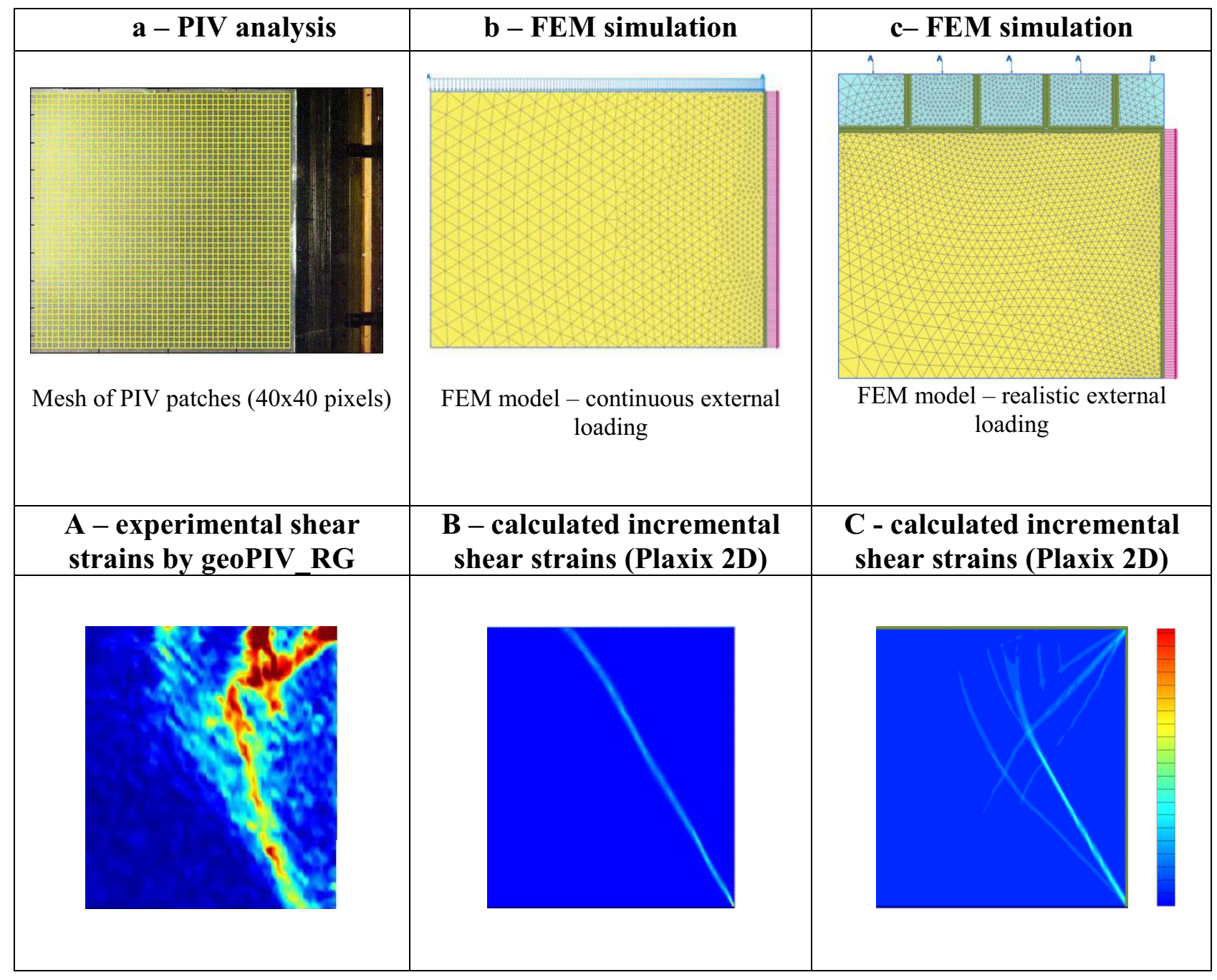

Fig. 2. Comparison of experimental (column A) and calculated (column B and C) shear strain fields evolved due to a vertical wall (right boundary) incremental horizontal displacement. 


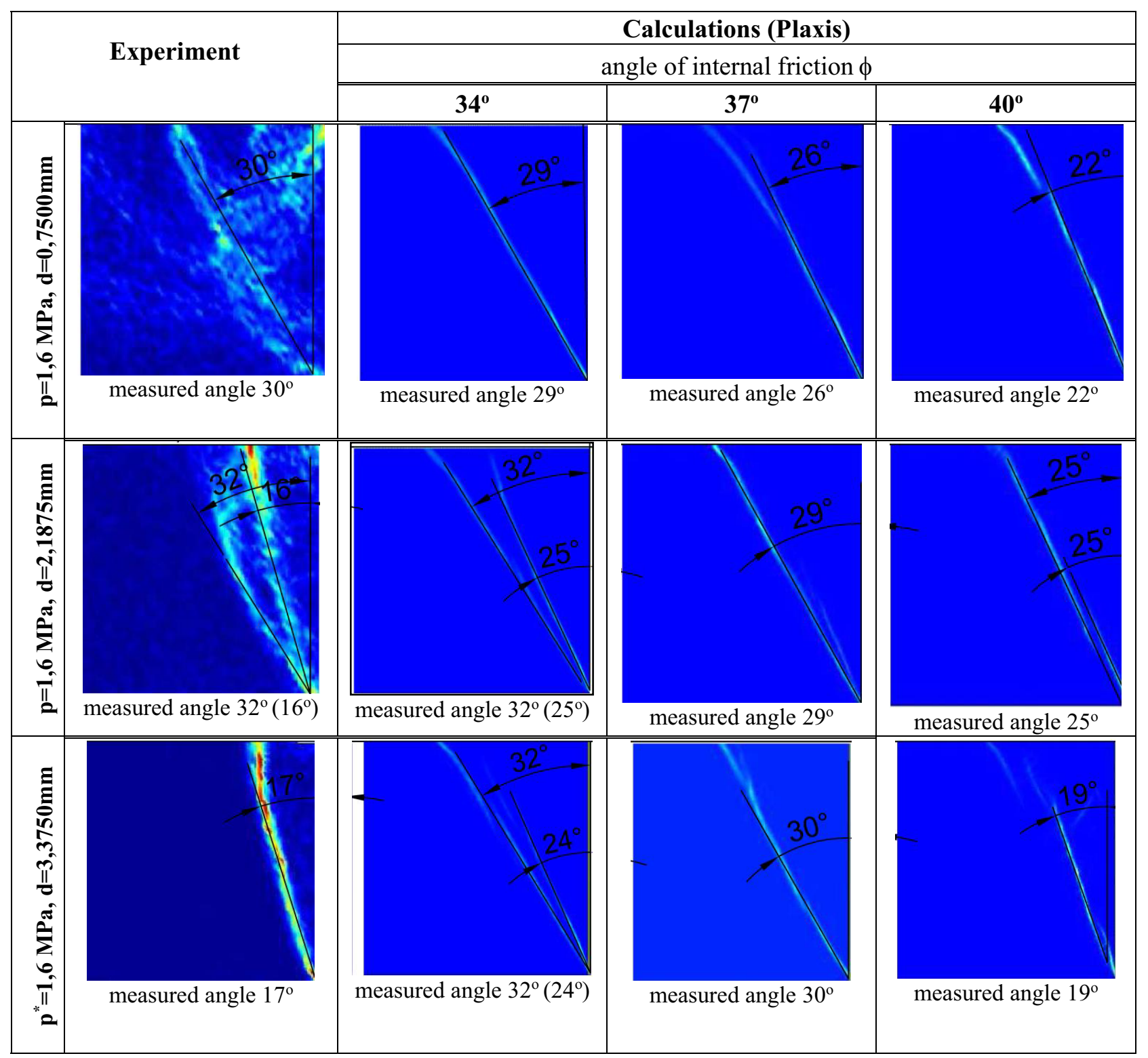

Fig. 3. Average inclination of strain localisations: experiment and FEM simulation. * - p - load, $d$ - displacement

\subsection{Materials}

Starlitbeads1000 spherical glass granules were used to form a granular sample $\left(\mathrm{d}_{50}=1.1 \mathrm{~mm}\right)$. Only dense samples were investigated. The glass granules were selected to represent soil due to their transparency.

The tests were registered both in ordinary and polarized light, to give not only strain, but also stress information based on photo-elasticity, but the latter is not a subject of this paper. Due to the demands of photoelastic method the granular specimen was saturated with clove oil, having the same refraction index as glass [4, 611]. Volume weight of granules was $16,80 \mathrm{kN} / \mathrm{m} 3$.

\subsection{Model tests}

Small scale model tests were performed on rectangular granular sample retained by a rigid wall. Deformation of the sample resulted from simple wall translation in the direction 'from the soil" (active earth pressure state).

The experimental setup and the techniques applied in this research were described earlier [4, 6-11].

The granular sample consisted of about $10^{6}$ spherical glass particles $(\sim 1 \mathrm{~mm}$ in diameter $)-$ a substitute material for sand.

The sample was tested in a plane strain apparatus, equipped with a loading system and rigid model wall able to translate horizontally. The granular material, placed in the box by pouring from a hopper, was statically loaded on the top surface and deformed due to incremental horizontal translation of right boundary. Model dimensions and arrangement are given in Fig. 1.

Small increment of the boundary translation was applied (less than 0.25 grain diameter) to capture momentary changes within strain fields.

All the images used in this research were taken by an industrial grade camera. 


\section{Numerical simulations by FEM}

The value of the stiffness modulus of glass granules was adopted based on triaxial compression test and was equal to $150000 \mathrm{kPa}$. For loading segments an elastic model of material was adopted and parameters for steel were accepted, from which they were made.

Maps of shear strain increments were selected to be presented, because they indicate best the locations of strains. Examples of such maps obtained for both types of loading are shown on Fig. 2B and 2C.

Since experimentally approximated internal friction angles ranged from $27^{\circ}$ to $34^{\circ}$, calculations were carried out for the slightly wider range $25^{\circ}-40^{\circ}$.

Specific boundary conditions - external load distributed on the sample through the set of five metal segments, Fig.1, [4, 6-11] arose the question of their influence on the deformation field. Therefore, a simulation of model test was carried out for two types of load distribution: direct (continuous load Fig. 2b) and applied through the five rigid segments, like it was in reality (Fig. 2c).

Figs $2 b$ and $2 c$ present the results of simulations for both versions of top boundary loading. It is clear that segmented loading (Fig.1) may promote multiple strain localisation. The arrangement of shear bands shown in Fig.2C do not depend on the mesh refinement (the width of shear bands does) and is very close to what was observed in some tests (Fig.2A).

The other issue addressed by numerical analysis was the inclination of strain localisations observed in model tests - it varied significantly as Fig.3 (Experiment) shows.

It can be seen from Fig.3 (Calculations) that the inclination of strain localisations depends on the value of internal friction angle in a way explaining any difference in shear bands inclination, observed experimentally.

\section{Conclusions}

The mechanisms of a granular material deformation are still not sufficiently recognized, even in case of such a simple test arrangement, like vertical retaining wall moving horizontally. Even simple Coulomb-Mohr model can be helpful in interpreting experimental findings in case of granular materials.

It was found that the general alignment of strain localization pattern (failure mechanism) may belong to macro scale features rather and be dominated by a test boundary conditions rather than the nature of the granular sample.

\section{Acknowledgments}

This work was done within the frame of the project nr.2011/03/B/ST8/05865, Polish National Science Centre.

\section{References}

1. D. J. White, W. A. Take, Technical Report DSOILS-TR322. Cambridge University Engineering Department (2002)

2. D. J White, D. J., W. A. Take, M. D. Bolton, Geotechnique 53, 619-631 (2003)

3. D. J White, M. Randolph, B. Thompson, Int. J. Phys. Model. Geotech. 3, 1-12 (2005)

4. D. Lesniewska, D. Muir Wood, Geotechnique, 60, doi: 10.1680/geot.2010.60.00.1 (2010)

5. O. C. Zienkiewicz, M. Huang, M. Pastor, Int. Jnl. For Numerical and Analytical Methods in Geomechanics, 19, 127-148 (1995)

6. D. Lesniewska, D. Muir Wood, J Eng Mech 135:1038-1054 (2009)

7. D. Muir Wood, D. Lesniewska, Granul Matt 13:395-415 (2011)

8. M. Pietrzak, D. Lesniewska, Studia Geotechnica et Mechanika, XXXIV, 4 : 69-77 (2012)

9. D. Lesniewska, M. Pietrzak, AIP Conf. Proc. 1542, 425-428 (2013)

10. D. Lesniewska, M. Pietrzak, Springer Series in Geomechanics and Geoengineering, 273-278 (2015)

11. D. Lesniewska, M. Pietrzak, J. Tejchman, Geomechanics from Micro to Macro, 1-2, 1025$1030(2015)$ 